\title{
Alteplase for Treating Thrombosis in Kawasaki Disease Patients with CAA Should Not be Limited to 12 Hours of Illness: A Single-Center Study Report
}

yanqiu chu

Shengjing Hospital of China Medical University

yunming $x u$

Shengjing Hospital of China Medical University

ce wang

Shengjing Hospital of China Medical University

xiaona yu

Shengjing Hospital of China Medical University

quanmei ma

Shengjing Hospital of China Medical University Department of Radiology

hong wang ( $\square$ wanghong_64@sina.com )

Shengjing Hospital of China Medical University https://orcid.org/0000-0002-0209-2899

Research article

Keywords: children, Kawasaki disease, coronary aneurysm, thrombosis, alteplase, heparin, warfarin, prognosis

Posted Date: February 22nd, 2022

DOI: https://doi.org/10.21203/rs.3.rs-1302952/v1

License: (c) (i) This work is licensed under a Creative Commons Attribution 4.0 International License. Read Full License 


\section{Abstract \\ Objective}

The most severe complication associated with giant coronary aneurysm in children with Kawasaki disease is ischemic cardiomyopathy (ICM) caused by thrombolysis. Effective thrombolytic therapy has a significant impact on patients' quality of life in long term.

\section{Methods}

To describe the effects of alteplase for once + heparin for 1 week/ then continue warfarin + aspirin + clopidogrel oral treatment for KD children with central thrombus in coronary aneurysm. These patients were treated in pediatric cardiology department of Shengjing hospital, China medical university, between 2020 Jan to 2021 August.

\section{Results}

Total 4 boys ( 7 times) had CAA complicated with central thrombosis. 5 times treatment with alteplase and heparin/ warfarin/ aspirin/ clopidogrel for 10 days to 2 months, all succeed.

\section{Conclusion}

1. In KD pediatric patients complicated with coronary aneurysm thrombosis, small but convexity lumen thrombus with strong echo less than one month and large thrombus with strong and weak mixed echo, coronary artery blood flow can be improved or completely recovered after the above treatment. 2 . Alteplase should not be strictly limited to within 12 hours of thrombosis. 3. Children with KD complicated with coronary aneurysm thrombosis are prone to thrombosis again.

\section{Full Text}

Pediatric Kawasaki disease has replaced rheumatic heart disease as the first acquired heart disease in developed countries even in China and in Indian [1][2][3]. According to the AHA 2004 and Japanese KD guidelines of the 5 th revised edition, CAA means coronary artery $\geq 4 \mathrm{~mm}$ or $Z$ score $\geq 5$. The most serious complication affecting the life quality in children with $\mathrm{KD}$ is the thrombosis in CAA [4]. Giant CAA is difficult to recover completely. It usually stays through childhood until adulthood when CAA needed to be treated. It has become a new challenge for pediatricians to treat thrombosis effectively as soon as possible, in order to maintain patient's normal cardiac function and normal development, and eliminate ICM development in KD children.

\section{Methods}


Total $4 \mathrm{KD}$ boys with thrombosis in CAA and convex to lumen were treated in Shengjing hospital affiliated with China medical university between 2020 Jan and 2021 May. Data including ECG, ECHO, and CTA were collected for comparison analysis on clinical symptoms, timeline and location of CAA and blood clots, and treatment methods and prognosis.

Inclusion criteria: i) symptoms and signs in the acute phase met the diagnostic criteria of KD/IKD [4]; ii) confirmed coronary aneurysms or giant aneurysm in coronary arteries using ECHO or coronary CTA at one of these time periods met the diagnostic criteria, according to KD Diagnostic Criterion (2002) of the Kawasaki Disease Research Committee of the Ministry of Health and Welfare of Japan [5], and guidelines issued by the American Heart Association (AHA) for the diagnosis of Kawasaki disease and atypical Kawasaki disease in 2017. iii) Convex lumen thrombosis in coronary aneurysms confirmed by ECHO or CTCA. Exclusion criteria: 1) No follow-up; 2) Without anticoagulant or thrombolytic therapy; 3) attached CAA wall without effects on blood flow.

\section{Treatment}

\section{Thrombolytic therapy}

Alteplase, at $1.5 \mathrm{mg} / \mathrm{kg}, 2$ hours intravenous infusion [6].

Anticoagulant therapy: A: Heparin 100U/kg, once 8 hours, intravenous injection (IV) [7]. B: Warfarin the first dose of $0.2 \mathrm{mg} / \mathrm{kg}$.d followed by next day of $0.1 \mathrm{mg} / \mathrm{kg}$.d orally, DIC was monitored and maintained INR between 2-3 [8].

Anti-platelet therapy: A: Aspirin 3-5mg/kg.d, orally for QD. B: Clopidogrel bisulfate1mg/kg.d, orally for QD, C: Dipyridamole 3-5mg/kg. D, divided into BID orally.

\section{Treatment method}

Method 1 to treat patients with giant CAA without thrombosis』warfarin+asprin+dipyridamole/clopidogrel.

Method 2 to treat patients with giant CAA+ small thrombosis $₫$ heperin+asprin+dipyridamole/clopidogrel 710days, then method 1 .

Method 3 to treat patients with giant CAA+giant thrombosis $\square$

Heperin 10U/kg iv, then Alteplase once+asprin+ dipyridamole/clopidogrel, then method 2.

\section{Statistic analysis:}

The different outcomes of method 1,2, and 3 were compared using descriptive methods.

\section{Results}


The average age of patients at the first visit was 4 years and 5 months (1.5-8 years) for 4 children (6 times) with KD complicated with central thrombus in CAA.

Total 4 patients (5 times) were treated with alteplase. Patient \# 1: ECHO presented mixed echo thrombosis in LAD at 2months of illness, and the thrombosis disappeared after thrombolytic/ anticoagulant/antiplatelet treatment for 2 months (Figure 1). Patient \#2, thrombosis formed twice in RCA. The second thrombosis blocked blood flow of distal RCA, and first disappeared after thrombolytic/anticoagulant/antiplatelet for 9 days, while the second times blood flow almost to pass after 2 months treatment (Figure 2). Patient \# 3, ECHO showed mixed thrombus (the strong echo was mixed with the weak echo) in LAD at 6.5 years of illness. After 9 days of thrombolytic/ anticoagulant/ antiplatelet treatment, the low density portion disappeared and his symptoms of chest tightness disappeared (Figure 3). Patient \# 4, ECHO showed LM aneurysmal dilatation filled with strong echo thrombosis $6.2 \star 2.6 \mathrm{~mm}$ at 15 days of illness. After treatment for 17 days, thrombosis disappeared (Figure 4). See Figure 1-4.

\section{Discussion}

Cardiovascular manifestations and complications are the major contributors to morbidity and mortality associated with KD, both during the acute illness phase and in the long-term progression [5]. Rupture of CAA in $\mathrm{KD}$ is rare. If the blood flow in CAA is unobstructed, the cardiac function will not be significantly impacted. In the subacute stage and recovery stage of KD, the elevated platelet count and platelet activity will increase the risk of thrombosis [9]. Meanwhile, KD children with CAA usually do not respond to IVIG treatment very well, and therefore will need repeated treatment with IVIG and glucocorticoids. Both drugs will increase platelet abnormally, increasing the risk of thrombosis [10-12]. The symptoms of ischemia are obvious in a short term and the time of occurrence can be clearly indicated in adults. Therefore, thrombolytic therapy can be managed within 12 hours of the onset. Thrombosis in children with Kawasaki disease occurs in dilated coronary aneurysms, and the formation process is slow and the possibility of complete occlusion in the short term is low. Therefore the symptoms are not as obvious as in adults [13]. In addition, it is difficult for young kids to accurately describe the specific time of ischemia symptoms. As a result, thrombolytic therapy has been rarely carried out and clinical experience of treatment is limited [4]. Currently, only 2 cases of 29-day-old [14] and 3-month-old infant [15] with coronary artery thrombosis have been reported being treated with alteplase in the United States. An adult $\mathrm{KD}$ was treated with combination of alteplase and actilyse for KD associated coronary aneurysm thrombosis [16]. At present, anticoagulation and antiplatelet are mainly used for thrombus treatment in world wide. However, the treatment data at our center suggests that these two methods have better results for preventing thrombosis than for treating existing thrombosis.

The treatment summary of our center is as follows: We did comparison of treatments in 2010 and 2015, a combination treatment of warfarin + aspirin + dipyridamole treatment was used to treat 2 misdiagnosed children with LAD thrombosis. At the end, 1 patient died and the other patient is disabled [17][supplementary file]. Similarly, the LAD was almost completely occluded in the first case. After the 
thrombolytic treatment with Alteplase, heparin was given for a week, followed by combination of warfarin + aspirin + clopidogre treatment, the thrombus disappeared completely 2 months later. In 2018, a case of distal RCA thrombosis was treated with heparin for 1 week, followed by combination of warfarin + aspirin + dipyridamole. There was no change in thrombosis observed 10 months later. CMR confirmed the formation of myocardial transmural necrosis in the inferior left ventricular wall [Figure 5]. It took 26 months for the thrombus to partially mechanize, but myocardial necrosis was irreversible. In the second similar case, blood flow was interrupted by a distal thrombosis of the RCA. After 2 months of treatment with Alteplase, then heparin for 1 week, followed by warfarin + aspirin + clopidogre treatment, blood flow in the distal trunk of the RCA was significantly recanalized. 2. For patients who have low density thrombosis, heparin IV for 7 days followed with warfarin plus antiplatelet therapy has a good prognosis (case 1 at the first times of thrombosis) [18]. 2. Thrombolytic therapy + heparin/warfarin + antiplatelet therapy should be performed with alteplase for large low-high density thrombus projecting to the lumen or affecting blood flow (case 1 second times/case 2 at the first times/case 3 at the second times/case 4 of thrombosis). Although the course of treatment is longer, the prognosis is good as long as the treatment persists. The side effects of alteplase in children are mainly bleeding in pursuance of pinhole and bleeding in the gingival area of caries, which usually occur within 1 hour of medication. Local cotton ball compression and halving the infusion speed of alteplase can stop the bleeding. Because heparin can lead to thrombocytopenia, blood routine should be monitored during heparin administration [19]. Most children didn't present with myocardial ischemia symptoms, which might be related to an adaption due to the slow formation, and also the restriction on vigorous activities by the child's parents. KD Patient \# 2 had myocarditis and was IVIG resistance. Thus, he was given IVIG $2 \mathrm{~g} / \mathrm{kg}$ for 2 times and oral glucocorticoids for one month, his platelet increased to $770 \times 10^{9} / \mathrm{L}$ at 19 days of illness. Thrombosis was found at 30 days after the disease, the platelets were recovered to $336 \times 10^{9} / \mathrm{L}$ while it was normal at 25 days of illness. 6 days later, alteplase treatment was performed and 10 days later, thrombosis diminished. This could be related to that platelet activity increased in the subacute stage and thrombosis is likely to be formed [3] [20-21]. Aspirin and dipyridamole, besides their anti-platelet activity, also reduce tissue factor procoagulant activity, and aspirin inhibits the STING pathway upstream of STING [22]. Patient \# 3 was a 1.5-year-old boy at onset, and warfarin, asprin, and dipyridamole were used to treat bilateral coronary aneurysms. The medication was not continuous. Hyperechoic thrombosis in the LAD was found 5.5 years after the onset of the disease (more than 1 year after the last ECHO examination). There was no change in thrombus after half a month of treatment with treated with haprin, warfarin, asprin, and dipyridamole. Myocardial ischemia after strenuous activity occurred 6.5 years after the onset of the disease. Mixed density thrombosis was still found in LAD. After treatment with alteplase and then continued with haprin, warfarin, asprin, and clopidogrel, the low-density part thrombus disappeared 2 weeks later, and his daily activity recovered. Interventional coronary thrombolysis has been established for adult patients. However, coronary artery openings in children are much narrower, and specialists prefer not to perform similar coronary thrombolysis procedures in infant patients [23-25]. In the United States, it has been reported that in the treatment of adult KD coronary thrombosis, thrombolysis should be performed in the coronary artery first, and if the thrombolysis is not successful, intravenous thrombolysis should be continued (the time is far longer than 12 hours, and the drug has been given for several times), 
which also achieves good results [17]. In Japan, it has been reported that intracoronary urokinase and post-thrombolytic regimen is successful in treating an infant patient with Kawasaki disease and acute myocardial infarction. Thus, it is possible to dissolve thrombus formed in the vascular lumen with treatment even if it occurred over 12 hours. But it takes a longer time to treat thrombosis formation lasting more than 12 hours [26].

To sum up, since adult coronary atherosclerosis can be accompanied by cerebral atherosclerosis and gastrointestinal ulcers, the biggest risk of thrombolysis is internal bleeding, and even thrombolytic therapy of pulmonary embolism is likely to cause bleeding [27]. However, after multivariate analysis, thrombolytic therapy using alteplase for myocardial infarction/cerebral infarction has not been associated with gastrointestinal tract or cerebral hemorrhage [28]. Children basically do not have cerebral atherosclerosis, and gastrointestinal ulcers are rare in them. So bleeding usually occurs in superficial area where it will be easily observed (eg. epistaxis, the site of skin puncture, caries oozing blood and so on). Therefore risk is relatively low when using thrombolytic therapy. In addition, coronary heart disease in adults occurred after coronary atherosclerosis stenosis lumen. The symptoms due to thrombus blocking blood flow are obvious, and the onset time can be captured clearly. In children with KD, thrombosis occurred within expanded aneurysm, and the thrombus grew relatively slow, rarely obstructs blood flow suddenly. It is also hard for young children to describe symptoms. Therefore it is difficult to manage the treatment within 12 hours of onset. In addition, the effect of anticoagulant and antiplatelet drugs alone is limited. Intravenous alteplase is an excellent remedy even after more than 12 hours the onset of symptoms. Therefore, in the case of KD complicated with coronary aneurysm thrombosis, our clinical experience in treating KD complicated with thrombus in coronary aneurysms is as follows: 1 . When Giant CAA mural thrombus does not affect blood flow, only anticoagulant and antiplatelet therapy is required. 2. For central thrombus in one of the following situations, as long as DIC and platelets are within the safe range, it is not necessary to strictly limit the occurrence time of thrombus $<12$ hours.

\section{Conclusion}

Alteplase should not be strictly limited to within 12 hours of thrombosis as long as DIC and platelets are within the safe range, coronary artery blood flow can be improved or completely recovered after the treatment. Children with KD complicated with coronary aneurysm thrombosis are prone to thrombosis again.

\section{List Of Abbreviations}

ICM: ischemic cardiomyopathy

KD: Kawasaki disease

IKD: incomplete KD

CAA: coronary artery aneurysm 
ECG: Electrocardiograph

ECHO: Echocardiograph

CTCA: computer tomography of coronary artery angiography

QD: once a day

BID: bis in die

LM: left main coronary artery

LAD: left anterior descending branch

LCX: left circumflex artery

RCA: right coronary artery

DIC: disseminated intravascular coagulation

CMR: Cardiac magnetic resonance

LVED Left ventricular end distolic diameter

LVEF Left ventricular ejection fraction

\section{Declarations}

\section{Ethical Approval and Consent to participate}

Study approval was obtained from the Ethics Committee of Shengjing Hospital affiliated to China Medical University. Case no:2021PS255J. Parents/legal guardians and patients older than 8 years of age signed the informed consent form before any study-related procedures occurred.

\section{Consent for publication}

Consent has been given by the patients and parents, via the written informed consent.

\section{Availability of supporting data}

The datasets used and analysis during the current study are available from the corresponding author on reasonable request.

\section{Competing interests}

The authors declare that they have no conflicts of interest. 


\section{Funding}

No Funding support the manuscript.

\section{Authors contributions}

$\mathrm{WH}^{\star}$ : patients diagnosis and treatment, images choosing, manuscript editor

CYQ: 3 patients treatment and observation, manuscript editor

XYM: 3 patients treatment and observation

WC: one patients treatment and observation

YXN: ECHO perform

MQM: CTCA perform

\section{Acknowledgements}

Thanks Dr. Xuemei Li, she did some observation with me to patient 2.

\section{Author informations}

1. Pediatric department of shengjing hospital, China Medical University, shenyang 110004, China

2. Ultrasonic department of shengjing hospital, China Medical University, shenyang 110004, China

3. Radiology department of shengjing hospital, China Medical University, shenyang 110004, China

\section{References}

1. Chen JJ, Ma XJ, Liu F, Yan WL, Huang MR, Huang M. et al. Epidemiologic features of Kawasaki disease in Shanghai from 2008 through 2012. Pediatr Infect DisJ.(2016), 35:7-12. doi:10.1097/INF. 0000000000000914.

2. Pilania RK, Singh S. Kawasaki Disease. In: Cimaz R, editors. Periodic and Non-Periodic Fevers. Rare Disease soft he Immune System. Cham: Springer (; 2020. pp. 45-63. doi:10.1007/978-3-030-190552_4.. In. : ).

3. McCrindle BW, Rowley AH, Newburger JW, Burns JC, Bolger AF, Gewitz M, et al Diagnosis, Treatment, and Long-Term Management of Kawasaki Disease: A Scientific Statement for Health Professionals From the American Heart Association. Circulation. (2017), 13:e927-99. doi:10.1161/CIR.0000000000000484.

4. Newburger JW, Takahashi M, Gerber MA, Gewitz MH, Tani LY, Burns JC, et al. Diagnosis, treatment, and long-term management of Kawasaki disease: a statement for health professionals from the Committee on Rheumatic Fever, Endocarditis, and Kawasaki Disease, Council on Cardiovascular 
Disease in the Young, American Heart Association. Pediatrics. 2004 Dec;114(6): 1708-33. doi: 10.1542/peds.2004-2182. Erratum in: Pediatrics. (2005),115(4):1118.

5. Ayusawa M, Sonobe T, Uemura S, Ogawa S, Nakamura Y, Kiyosawa N,et al. Revision of diagnostic guidelines for Kawasaki disease (the 5th revised edition). Pediatr Int (. 2005;47(2):232-4. doi:10.1111/j.1442-200x.2005.02033.x.

6. Wang H, Zhang Y, Hou Y, Yu X. Misdiagnosed KD. In: Wang H, editor. Paediatric Kawasaki Disease. Singapore: Springer; 2021. https://doi.org/10.1007/978-981-15-0038-1_12.

7. TKobayashi T, Ayusawa M, Suzuki H, Abe J, Ito S, Kato T, et al. Revision of diagnostic guidelines for Kawasaki disease (the 6th revised edition) [J]. 62: Pediatrics International; 2020. pp. 1135-8. DOI:10.1111/ped.14326.

8. Cannon CP, Gibson CM, McCabe CH, Adgey AA, Schweiger MJ, Sequeira RF, et al. TNK- tissue plasminogen activator compared with front-loaded alteplase in acute myocardial infarction: results of the TIMI 10B trial. Thrombolysis in Myocardial Infarction (TIMI) 10B Investigators.Circulation. (1998), 98:2805-14. doi: 10.1161/01.cir.98.25.2805.

9. Gosselin RC, King JH, Janatpour KA, Dager WE, Larkin EC, Owings JT. Comparing direct thrombin inhibitors using aPTT, ecarin clotting times, and thrombin inhibitor management testing. Ann Pharmacother. 2004;38:1383-8. doi:10.1345/aph.1D565.

10. Fayyaz M, Abbas F, Kashif T. The Role of Warfarin and Rivaroxaban in the Treatment of Cerebral Venous Thrombosis. Cureus. 2019;11:e4589. doi:10.7759/cureus.4589.

11. Shenkman B, Matetzky S, Fefer P, Hod H, Einav Y, Lubetsky A, et al. Variable responsiveness to clopidogrel and aspirin among patients with acute coronary syndrome as assessed by platelet function tests. Thromb Res (2008), 122:336-45. doi: 10.1016/j.thromres. 2007.10.018.

12. Ying-Ying Z, Ting-Ting W, Yi Y, Xian-Geng H, Ying G, You C, et al. Personalized antiplatelet therapy guided by a novel detection of platelet aggregation function in stable coronary artery disease patients undergoing percutaneous coronary intervention: a randomized controlled clinical trial. Eur Heart J Cardiovasc Pharmacother. (2020), 6:211-21. doi: 10.1093/ehjcvp/ pvz059.

13. Park JH, Choi HJ. Clinical implications of thrombocytosis in acute phase Kawasaki disease.Eur $\mathrm{J}$ Pediatr. (2021), Feb 1. doi: 10.1007/s00431-021-03966-8. Online ahead of print.

14. Nishino S, Kodaka T, Sawada Y, Goka T, Gotoh Y, Tsunemine H, et al. Marked rebound thrombocytosis in response to glucocorticoids in a patient with acquired amegakaryocytic thrombocytopenia. J Clin Exp Hematop. (2018), 58:166-70. doi: 10.3960/jslit. 18016.

15. Lo JY, Minich LL, Tani LY, Wilkes J, Ding Q, Menon SC. Factors Associated With Resource Utilization and Coronary Artery Dilation in Refractory Kawasaki Disease (from the Pediatric Health Information System Database). Am J Cardiol. (2016),118:1636-40. DOI: 10.1016/ j.amjcard.2016.08.039.

16. Jone PN, Tapia D, Davidson J, Fagan TE, Browne L, Ing RJ, Kay J. Successful Treatment of Myocardial Infarction in an Infant With Kawasaki Disease. Semin Cardiothorac Vasc Anesth. 2015;19:255-9. doi:10.1177/1089253215573328. 
17. Vijay R, Karia GC, Hescock A, Gedalia, et al. Successful emergent coronary thrombolysis in a neonate with Kawasaki's disease. Pediatr Cardiol. 2010;31:1239-42. doi:10.1007/s00246-010-9781-y.

18. Chandwaney RH, Stathopoulos T, Sunew J, et al Adjunctive therapies in the cath lab. Successful thrombolysis using the combination of tissue plasminogen activator and abciximab in an adult with Kawasaki's disease. J Invesive cardil. (2001),13:651-3. PMID: 11533505.

19. Wang H, Zhang Y, Hou Y, Yu X. Misdiagnosed KD. In: Wang H, editor. Paediatric Kawasaki Disease. Singapore: Springer; 2021. https://doi.org/10.1007/978-981-15-0038-1_12.

20. XuY-m ChuY-q, Wang LiX-m, MaQ-m C, Yu X-n, Yu X-y, Chen R, Xing Y-I, Yu X-x, Sun L, Cui X-z, Wang $\mathrm{H}$. The Complementary Relationship Between Echocardiography and Multi-Slice Spiral CT Coronary Angiography in the Diagnosis of Coronary Artery Thrombosis in Children With Kawasaki Disease. Front Pediatr 9:670887. 2021. doi:10.3389/fped. 2021.670887.

21. Greinacher A, Selleng K, Warkentin TE. Autoimmune heparin-induced thrombocytopenia. J Thromb Haemost. 2017 Nov;15(11):2099-114. doi:10.1111/ jth.13813. Epub 2017 Sep 28.

22. Arora K, Guleria S, Jindal AK, Rawat A, Singh S. Platelets in Kawasaki disease: Is this only a numbers game or something beyond? Genes Dis. (2019). PMID:32181276. https://doi.org/ 10.1016/j.gendis.2019.09.003.

23. Yahata T, Suzuki C, Yoshioka A, Hamaoka A, lkeda K. Platelet activation dynamics evaluated using platelet-derived microparticles in Kawasaki disease. Circ J. 2014;78:188-93. doi:10.1253/circj.cj-121037. Epub 2013 Oct 22.

24. Berthelot JM, Drouet L, Lioté F. Kawasaki-like diseases and thrombotic coagulopathy in COVID- 19: delayed over-activation of the STING pathway? Emerg Microbes Infect. 2020 Dec;9(1):1514- 22. doi: 10.1080/22221751. 2020.1785336.

25. Nakagawa M, Watanabe N, Okuno M, Okamoto N, Fujino H. Effects of intracoronary tissue-type plasminogen activator treatment in Kawasaki disease and acute myocardial infarction. Cardiology. 2000;94:52-7. doi:10.1159/000007046.

26. Horigome H, Sekijima T, Miyamoto T. Successful thrombolysis with intracoronary adminis- tration of tissue plasminogen activator in an infant with Kawasaki disease. Heart. 1997;78:517-8. doi:10.1136/hrt.78.5.517.

27. Tsubata S, Ichida F, Hamamichi Y, Miyazaki A, Hashimoto I, Okada T. Successful throm- bolytic therapy using tissue type plasminogen activator in Kawasaki disease. Pediatr Cardiol. (19 95),16:186-9.doi: 10.1007/BF00794192.

28. Katayama F, Hiraishi S, Takeda N, Misawa H. Intracoronary urokinase and post-thrombolytic regimen in an infant with Kawasaki disease and acute myocardial infarction. Heart. 1997 Dec; 78(6):621-2. doi: 10.1136/hrt.78.6.621.

29. Qiukui H, Bi Rong D, Jirong Y, Taixiang W, Guan JL. Thrombolytic therapy for pulmonary embolism.Cochrane Database Syst Rev. (2018),12: CD004437. Published online (2018) Dec 18. doi: 10.1002/14651858.CD004437.pub5. 
30. Matsuo R, Kamouchi M, Fukuda H, Hata J, Wakisaka Y, Kuroda J, et al Intravenous Thrombolysis with Recombinant Tissue Plasminogen Activator for Ischemic Stroke Patients over 80 Years Old: The Fukuoka Stroke Registry. PLoS One. (2014), 9: e110444. Published online 2014 Oct 16. doi: 10.1371 /journal. pone.0110444.

\section{Figures}

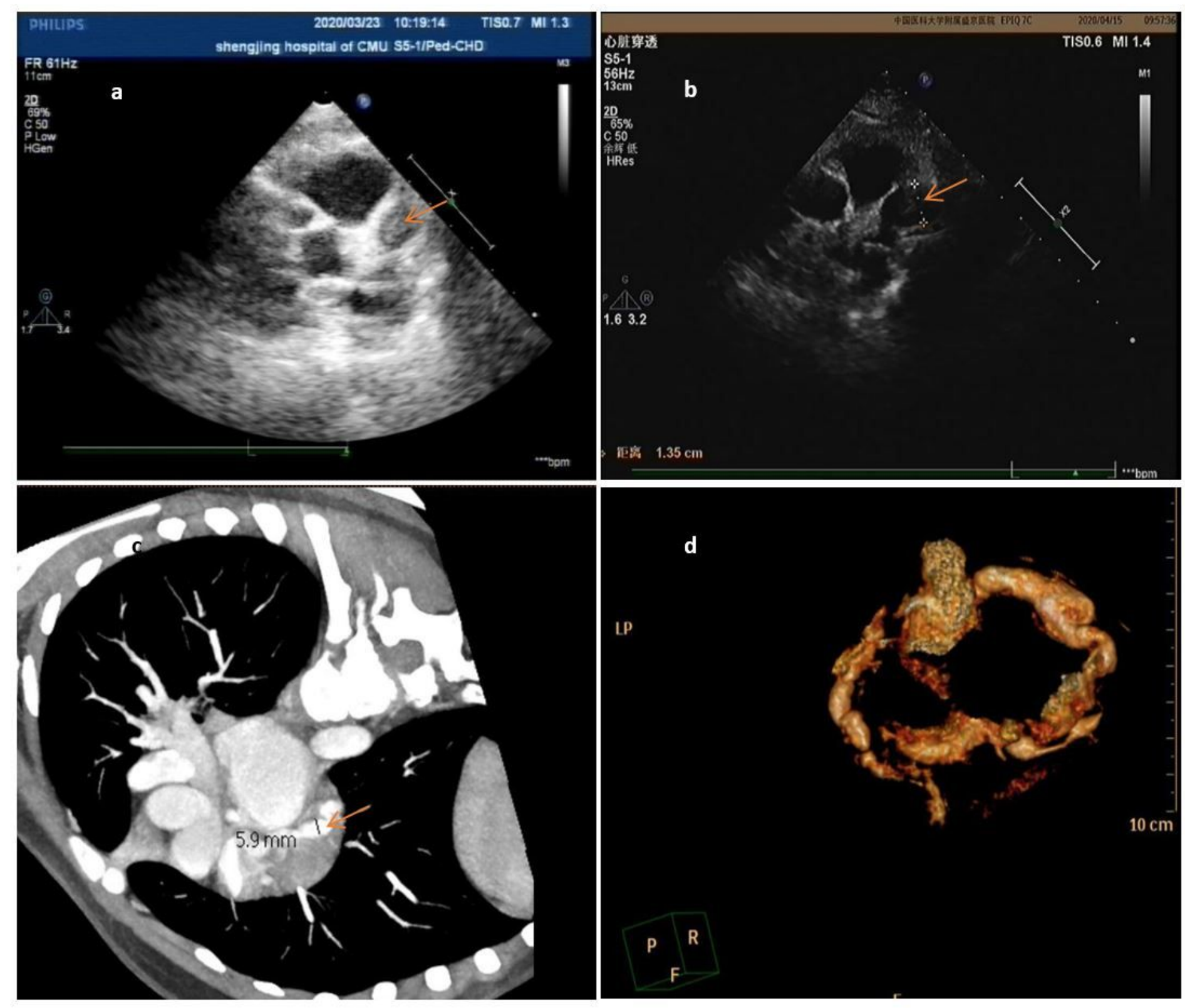

Figure 1

ECHO showed the thrombus formed in the LAD and the lumen was nearly blocked (a) at 2 months after the onset of the disease in Case 1. After 2 months of thrombolysis, the thrombus disappeared completely (b). No contrast filling defect (c) was found in the LAD in CTCA the day after the thrombosis. Bilateral coronary arteries showed irregular and marked dilation (d). 


\section{Figure 2}

ECHO showed RCA tumor-like expansion to $9 \mathrm{~mm}$ at 16 days of illness in case 2(a). At 30 days of illness, thrombus with relatively high density $(2.6 * 3.2 \mathrm{~mm}$, b-red arrow) was found and convex to the lumen in RCA (b). The treatment began six days later and the thrombus disappeared after 10 days treatment (c). Coronary artery CTA showed aneurysm in RCA with good distal blood flow at 30 days of illness (d). Thrombosis presented at the distal end of RCA when retested with CTCA at a different hospital at 17 months of illness, it blocked the blood flow (e). The distal blood flow almost recovered after 2 months treatment (f).

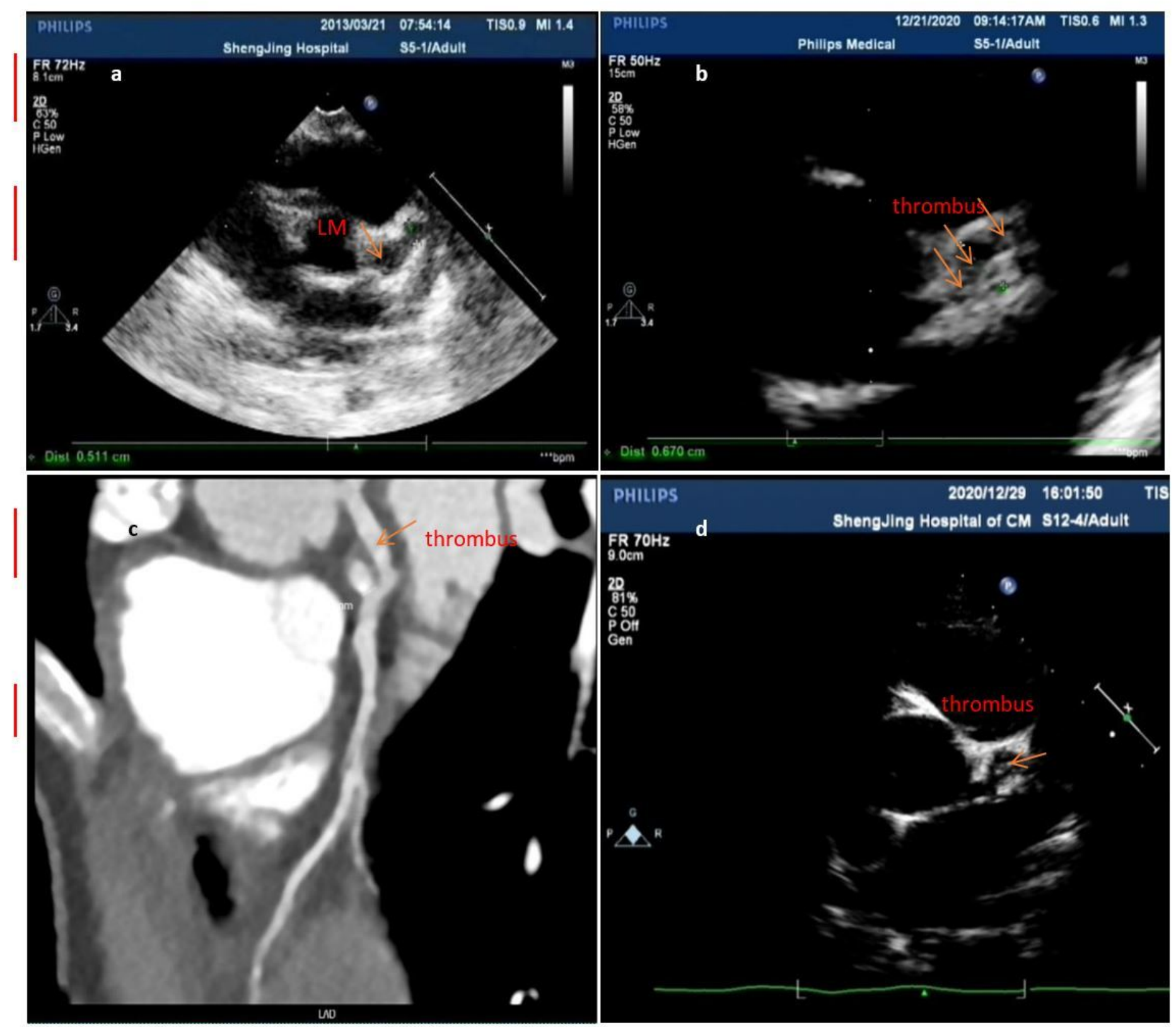

Figure 3 
ECHO showed LAD aneurysmal dilatation at 14 days of illness in case 3 (a). At 6.5 years of illness, ECHO showed mixed thrombus (the strong echo was mixed with the weak echo) in LAD (b). CTCA showed LAD dilatation with contrast agent filling defect (thrombus) (c). After 9 days of thrombolytic/anticoagulant/antiplatelet treatment, the low density portion (the newly formed thrombus) disappeared and patient's symptoms of chest tightness disappeared (d).

\section{Figure 4}

ECHO showed LM aneurysmal dilatation and filling with strong echo thrombosis $6.2 * 2.6 \mathrm{~mm}$ at 15 days of illness in case 4 (a). Treatment started with alteplase. 3days later ECHO still showed thrombus in LM (b). Contrast filling defect wasn't observed in CTCA (c) and at same time ECHO showed a small thrombus in LM(d) at 22 days of illness. ECHO showed that the thrombus was reduced to $2.3^{\star} 1.9 \mathrm{~mm}$ at 27 days of illness (e) and diminished at 32 days of illness (f). 

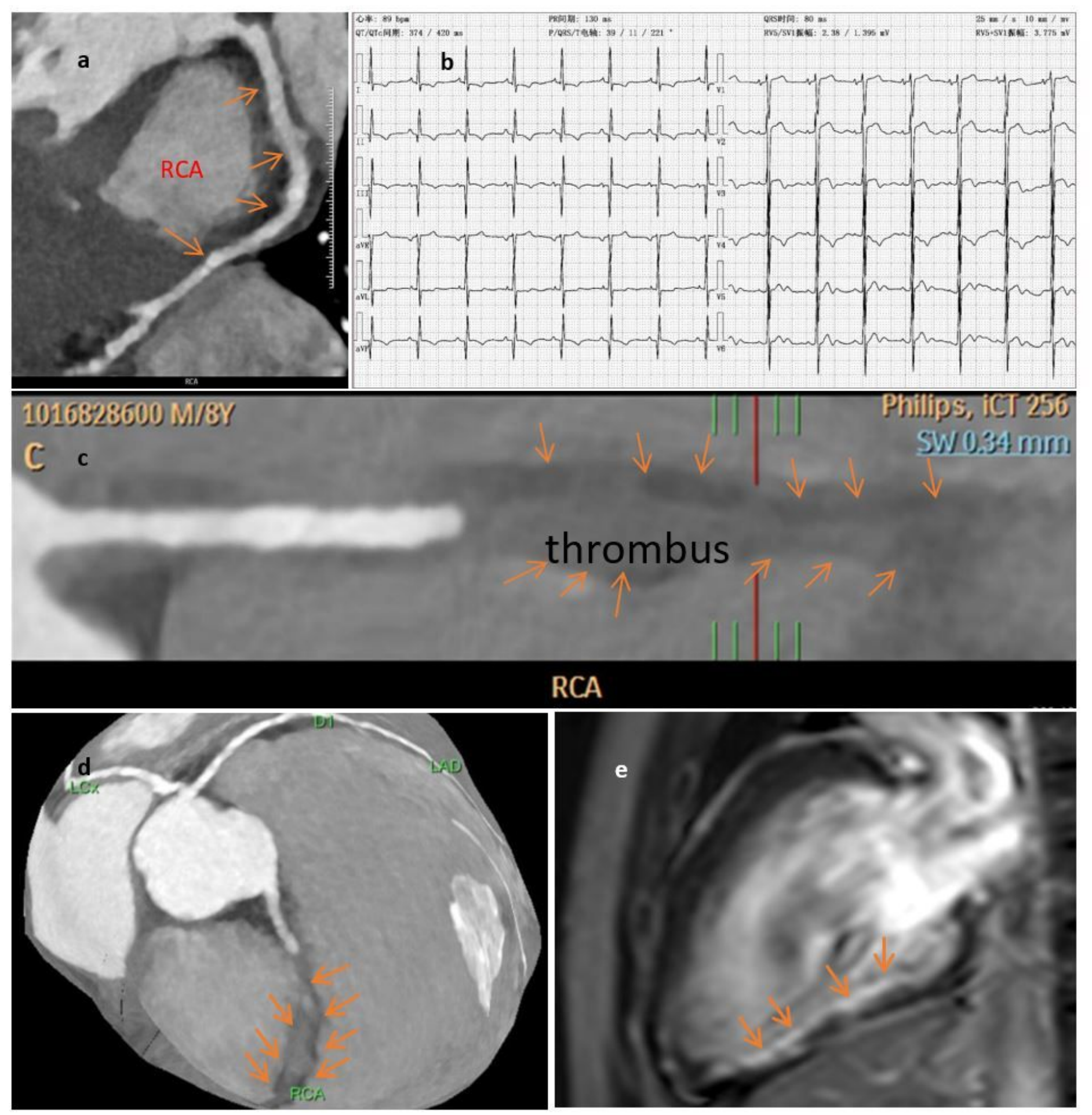

Figure 5

CTCA showed RCA mild dilatation and blood flow (a) at 20 days of illness in case 3 . Six months later, the T waves in the lower and lateral walls of the heart are inverted or bidirectional (b), CTCA confirmed distal RCA thrombosis occluding the vessel (c). he were treated with method 2. At 1.5 yeas of illness, CTCA showed distal RCA thrombosis still occluding the vessel (d), and CMR indicated left ventricular transmural necrosis (e). 


\section{Supplementary Files}

This is a list of supplementary files associated with this preprint. Click to download.

- Supplementary.docx 